\section{Ascitis posterior a trasplante renal pediátrico. Reporte de un caso}

\author{
Zaltzman-Girshevich $\mathrm{S}^{1}$, Sánchez-García $\mathrm{C}^{2}$, Suárez-Barajas EM², Corcuera- \\ Delgado $\mathrm{CT}^{3}$
}

\begin{abstract}
Resumen
La ascitis posterior a un trasplante renal es poco común y generalmente es causada por complicaciones en la técnica quirúrgica. Presentamos el caso de un niño de 5 años de edad con diagnóstico de glomerulonefritis crónica que recibió un trasplante renal de donador vivo relacionado con función adecuada del injerto durante las primeras horas de evolución; 72 horas después de la cirugía, desarrolló ascitis con un gradiente albúmina sérica-ascitis de 0.92 g/ dL. Debido a esto fue necesaria la exploración quirúrgica durante la cual se encontró ascitis en gran cantidad en la cavidad peritoneal con infiltración de líquido entre la cápsula y la superficie del parénquima del riñón nativo; se practicó nefrectomía de este riñón. En el examen histopatológico se observó dilatación acentuada de los vasos linfáticos. La evolución postquirúrgica del paciente fue satisfactoria. La ascitis quilosa se debe al drenaje de linfa hacia la cavidad peritoneal. En la literatura encontramos algunos casos de ascitis de origen linfático después de un trasplante renal. De acuerdo con los hallazgos descritos consideramos que en nuestro caso la ascitis se debió a trasudado de linfa desde la cápsula del riñón nativo, secundario a obstrucción de los vasos linfáticos por manipulación aórtica excesiva durante la cirugía. En pacientes pediátricos sometidos a trasplante renal intraperitoneal que desarrollan ascitis el origen linfático de ésta debe ser considerado.
\end{abstract}

PALABRAS CLAVE: ascitis quilosa, glomerulonefritis colapsante, nefrocalcinosis, trasplante renal, reporte de caso.

Acta Pediatr Mex. 2016 Nov;37(6):328-332.

\section{Ascites after pediatric renal transplant. Case report.}

Zaltzman-Girshevich $\mathrm{S}^{1}$, Sánchez-García $C^{2}$, Suárez-Barajas EM², CorcueraDelgado $\mathrm{CT}^{3}$

\begin{abstract}
The presence of ascites after a renal transplant is an uncommon entity generally caused by complications of the surgical technique. We present a 5 year-old male with diagnosis of chronic glomerulonephritis; who received a kidney transplant of a related live donor, with adequate function of the allograft during the first hours after transplant, developing ascites 72 hours after surgery, with a serum-ascites albumin gradient
\end{abstract}

${ }^{1}$ Profesor Titular de Nefrología Pediátrica. ${ }^{2}$ Médico Residente del Curso de Nefrología Pediátrica.

${ }^{3}$ Médico Adscrito al Departamento de Anatomía Patológica.

Instituto Nacional de Pediatría, México.

Recibido: 1 de marzo del 2016

Aceptado: 28 de junio del 2016

Correspondencia

Carlos Sánchez García

sanchez.carlos8516@gmail.com

Este artículo debe citarse como Zaltzman-Girshevich S, Sánchez-García C, SuárezBarajas EM, Corcuera-Delgado CT. Ascitis posterior a trasplante renal pediátrico. Reporte de un caso. Acta Pediatr Mex. 2016;37(6):328-332. 
of $0.92 \mathrm{~g} / \mathrm{dL}$. Because of this a surgical intervention was necessary; there was ascites in great amount in the peritoneal cavity and infiltration of liquid in between the surface of the native kidney and its capsule; nephrectomy of this kidney was done. In the histopathological exam severe dilatation of the lymphatic vessels was observed. The patient evolution after the surgery was satisfactory. Chylous ascites is due to the drain of lymph into the peritoneal cavity. In the literature we found some cases of ascites of lymph origin after kidney transplant. According to the findings described; we considered that the ascites was due to lymph transudate from the capsule of the native kidney secondary to obstruction of the lymph vessels due to excessive aortic manipulation during renal transplant surgery. In pediatric patients that have undergone an intraperitoneal renal transplant and develop ascites, the lymphatic origin of this should be consider.

KEYWORDS: chylous ascites; collapsing glomerulonephritis; nephrocalcinosis; kidney transplant; case report

\begin{abstract}
${ }^{1}$ Profesor Titular de Nefrología Pediátrica. ${ }^{2}$ Médico Residente del Curso de Nefrología Pediátrica.

${ }^{3}$ Médico Adscrito al Departamento de Anatomía Patológica.
\end{abstract}

Instituto Nacional de Pediatría, México.

Correspondence

Carlos Sánchez García

sanchez.carlos8516@gmail.com

\section{INTRODUCCIÓN}

Las complicaciones posteriores a un trasplante renal podemos dividirlas en médicas: infecciones, rechazo, toxicidad medicamentosa, etcétera, y quirúrgicas: vasculares, urinarias, linfáticas o que involucran la herida quirúrgica. ${ }^{1}$ La ascitis es un problema poco frecuente después de un trasplante renal; comúnmente las causas más relacionadas son las complicaciones quirúrgicas. Ésta es una condición que retrasa la recuperación del paciente. ${ }^{2}$ Se reporta el caso de un paciente que desarrolló ascitis en el postoperatorio inmediato y el reto diagnóstico que representó.

\section{REPORTE DE CASO}

Niño de 5 años de edad, $14.7 \mathrm{~kg}$ de peso y $104 \mathrm{~cm}$ de talla con diagnóstico de enfermedad renal crónica de etiología glomerular. Antecedente de síndrome nefrótico desde los 2 años de edad con mala respuesta al tratamiento debido a pobre apego al mismo. La biopsia renal mos- tró glomerulonefritis crónica avanzada (riñón terminal). El niño fue sometido a un trasplante renal anticipado de donador vivo relacionado, el aloinjerto fue colocado del lado derecho, intraperitoneal, con anastomosis de la vena y arteria renales a la vena cava inferior y a la aorta, respectivamente.

El paciente evolucionó satisfactoriamente con función adecuada del injerto renal; creatinina sérica inmediata de $6.3 \mathrm{mg} / \mathrm{dL}$ posterior a la cirugía (previa de $8.6 \mathrm{mg} / \mathrm{dL}$ ). En las primeras 24 horas con una creatinina sérica de $0.88 \mathrm{mg} / \mathrm{dL}$ disminuyendo hasta $0.78 \mathrm{mg} / \mathrm{dL}$ en las siguientes 48 horas. El tratamiento inmunosupresor utilizado fue prednisona, ciclosporina y azatioprina, así como amlodipino como antihipertensivo.

Tres días después de la cirugía el paciente tuvo disminución del volumen urinario, distensión abdominal y ligero incremento en la creatinina sérica $(1.0 \mathrm{mg} / \mathrm{dL})$. Se efectuó un ultrasonido Doppler abdominal que mostró líquido libre en cavidad peritoneal y adecuado flujo sanguíneo 
del injerto renal con índice de resistencias vasculares normal.

Se practicó cirugía exploratoria encontrando líquido libre en cavidad abdominal; aproximadamente $800 \mathrm{~mL}$, inodoro pero turbio con una cuenta leucocitaria de 21 células por microlitro; $60 \%$ de linfocitos; albúmina de $1.080 \mathrm{~g} / \mathrm{dL}$; con una albúmina sérica en ese momento de $2.0 \mathrm{~g} /$ dL; calculándose un gradiente de albúmina sérica-ascitis (SAAG) de $0.92 \mathrm{~g} / \mathrm{dL}$. Las anastomosis vasculares y el uréter del injerto se encontraron sin alteraciones; sin embargo, se observó formación persistente de líquido en el fondo de la cavidad peritoneal con infiltración de líquido de la cápsula del riñón nativo derecho, decidiéndose realizar nefroureterectomía. En el análisis histopatológico de este riñón se encontró dilatación de los vasos linfáticos tanto en el intersticio como en el hilio renal, así como podocitopatía de tipo focal y segmentaria, variedad colapsante con cicatrización avanzada, siendo esta la causa de la insuficiencia renal; además de depósitos corticales de calcio (nefrocalcinosis) (Figura 1).

Después de la cirugía el paciente evolucionó favorablemente con volumen urinario adecuado, normalización de la función del injerto (disminución de la creatinina sérica de 0.98 a $0.33 \mathrm{mg} /$ dL) y disminución progresiva de la ascitis. El paciente fue egresado asintomático.

\section{DISCUSIÓN}

La ascitis se define como la excesiva acumulación de líquido en la cavidad peritoneal. ${ }^{3}$ La ascitis quilosa es una complicación postquirúrgica rara, definida como el drenaje de linfa a la cavidad peritoneal, debido a daño u obstrucción del sistema linfático. ${ }^{4}$ En el caso de nuestro paciente no contamos con niveles de triglicéridos en el líquido peritoneal y la cuenta de leucocitos se encontró en menos de 500 células; sin embargo, podríamos considerar el origen linfático de

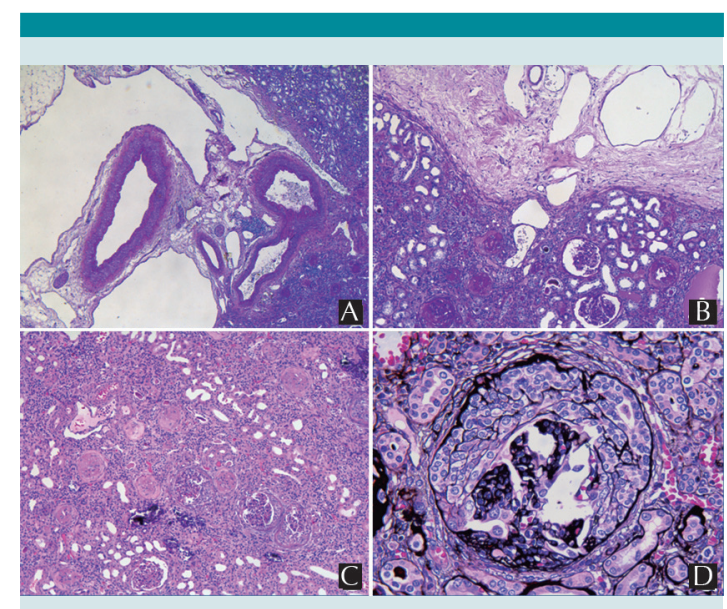

Figura 1. Estudio histopatológico del riñón nativo derecho. A) Tinción de PAS (4X): vista panorámica del hilio renal que exhibe dilatación vascular linfática acentuada. B) Tinción de PAS (10X): vista a mediano aumento que exhibe dilatación vascular linfática acentuada en la cápsula y en el parénquima de la corteza renal. C) Tinción de hematoxilina-eosina (4X): además de mostrar glomeruloesclerosis global difusa muestra glomérulos con podocitopatía focal y segmentaria, variedad colapsante, calcificación tubular e infiltrado inflamatorio linfocitario en el intersticio. D) Metenamina de plata (40X): glomerulopatía colapsante; son visibles la retracción del ovillo glomerular, el estrechamiento de las luces capilares, la proliferación y tumefacción de las células epiteliales viscerales.

la ascitis como posibilidad diagnóstica ya que el gradiente albumina sérica-ascitis se encontró debajo de $1.1 \mathrm{~g} / \mathrm{dL}^{5}$

Existen pocos reportes en la literatura de ascitis de origen linfático posterior a trasplante renal. En 1976 se reportó en Ontario, Estados Unidos, una mujer de 46 años quien 3 años después de un trasplante renal presentó líquido libre peritoneal claro trasudando de la cápsula del injerto. ${ }^{6}$

Así mismo, en 2004, en el Reino Unido, se reportó una paciente de 29 años postrasplantada renal con disfunción del injerto 10 meses des- 
pués de la cirugía; se practicó biopsia renal y se encontró microangiopatia trombótica secundaria al uso de tacrolimus; dos semanas después fue readmitida por ascitis; se describió durante la exploración quirúrgica un injerto renal grande trasudando fluido claro hacia el peritoneo; se consideró como causa de la ascitis un linfocele subcapsular drenando aparentemente por el sitio de punción de la biopsia renal. ${ }^{7}$

En otro reporte, en un paciente de 54 años con ascitis quilosa, se consideró que la etiología era una interrupción de los canales linfáticos o la fenestración de linfoceles postquirúrgicos. ${ }^{8}$

En pacientes pediátricos, encontramos tres casos reportados de ascitis quilosa posterior a trasplante renal: una niña de 7 años que presentó esta condición una semana posterior al evento quirúrgico y se relacionó a una extraordinaria manipulación de la aorta durante la cirugía y dos pacientes de 7 y 2.5 años que la desarrollaron dos meses y dos semanas después del trasplante, respectivamente. ${ }^{9,10}$

La presencia de ascitis en el postrasplante renal generalmente se ha relacionado con fuga de orina hacia la cavidad peritoneal. ${ }^{10} \mathrm{En}$ el paciente que presentamos no hay evidencia de composición urinaria en el líquido ascítico (la concentración sérica de creatinina fue casi idéntica a la concentración de creatinina en el líquido de ascitis). De forma similar a lo reportado en pacientes adultos describimos infiltración y trasudado de líquido por la cápsula renal; sin embargo, en este caso en el riñón nativo ipsilateral al injerto. En el estudio histopatológico se observó dilatación de los vasos linfáticos a nivel del hilio, siendo una posibilidad de esto la obstrucción del sistema linfático. Al igual que en los casos pediátricos reportados el trasplante se realizó intraabdominal y la anastomosis vascular se realizó a la vena cava inferior y la aorta, por lo que concordamos con la relación de esta patología y la manipulación de los grandes vasos y linfáticos adyacentes durante el procedimiento quirúrgico.

La presencia de dilatación de los vasos linfáticos se ha descrito también cuando hay obstrucción del drenaje venoso del riñón afectado. ${ }^{11}$

Es importante mencionar que como hallazgo durante el estudio de la nefrectomía del riñón nativo de nuestro paciente se encontró nefrocalcinosis, así como glomeruloesclerosis focal y segmentaria, variedad colapsante.

En la revisión de la literatura se encontró reportado un paciente masculino de 13 años de edad con glomerulonefritis crónica; con evidencia radiológica de nefrocalcinosis. ${ }^{12}$ No encontramos reportes de nefrocalcinosis en pacientes con ascitis postrasplante renal.

Según los hallazgos transquirúrgicos y anatomopatológicos descritos en nuestro paciente consideramos que la formación de ascitis fue secundaria a trasudado de linfa desde la cápsula del riñón nativo por obstrucción linfática, debida a manipulación aórtica excesiva durante el trasplante renal ya que, en los hallazgos histopatológicos, se corroboró dilatación linfática acentuada en el hilio y en el parénquima del riñón nativo.

La ascitis de origen linfático continúa siendo una causa rara de ascitis postrasplante renal, por lo que debemos considerarla en pacientes pediátricos sometidos a un procedimiento de trasplante intraperitoneal y anastomosis de la vena renal a la vena cava y de la arteria renal a la aorta.

En el caso presentado no existieron datos totalmente concluyentes para diagnosticar una ascitis quilosa, ya que no contamos con niveles de triglicéridos y el conteo de leucocitos se encuentra menor de 500; sin embargo, consideramos que el caso es Ilamativo porque la acumulación excesiva de líquido (ascitis) debido al drenaje 
de linfa a la cavidad peritoneal por daño del sistema linfático es infrecuente y no encontramos referencia reportada de esta entidad en pacientes pediátricos postrasplantados en México.

\section{REFERENCIAS}

1. Humar A, Matas AJ. Surgical complications after kidney transplantation. Semin Dial. 2005;18(6):505-10.

2. Markov M, Van Thiel DH, Nadir A. Ascites and kidney transplantation: case report and critical appraisal of the literature. Dig Dis Sci. 2007;52(12):3383-8.

3. Saif MW, Siddiqui IA, Sohail MA. Management of ascites due to gastrointestinal malignancy. Ann Saudi Med. 2009;29(5):369-367.

4. Al-Busafi SA, Ghali P, Deschênes M, Wong P. Chylous ascites: Evaluation and Management. ISRN Hepatology. 2014. DOI $10.1155 / 2014 / 240473$.

5. Cardenas A, Chopra S. Chylous ascites. Am J Gastroenterol. 2002;97(8):1896-900.
6. Clark WF, Sullivan SN, Lindsay RM, Wang YL, Turnbull ID, Linton AL. Ascites Secondary to Transudation from A Renal Cadaveric Transplant. JAMA. 1976;235(6):635.

7. Kulkarni S, Burns A, Al-Akraa M. Severe ascites following renal transplant biopsy caused by a rupture of a subcapsular lymphocele: treated successfully by retroperitonealization. Nephrol Dial Transplant. 2004;19(4):1022-3.

8. Liu WC, Kuo MC, Wu WJ, Hwang SJ, Chen HC. Chylous ascites after renal transplantation - a case report. Nephrol Dial Transplant. 2006;21(11):3336-7.

9. Itoh K, Tanda K, Kato C, Kanagawa K, Seki T. Intraperitoneal leakage of technectium-99m-DTPA following renal transplantation: a sign of chylous ascites. J Nucl Med. 1994;35(1):93-4.

10. Riar S, Warshaw B, Amaral S. Chylous ascites complicating pediatric renal transplantation. Pediatr Nephrol. 2012;27(8):1397-9.

11. Heptinstall RH. Pathology of the kidney. 2nd ed. Boston: Little, Brown; 1974. 539 p.

12. Cohen HL, Kassner EG, Haller JO. Nephrocalcinosis in chronic glomerulonephritis: report of the youngest patient. Urol Radiol. 1981;2(1):51-2.

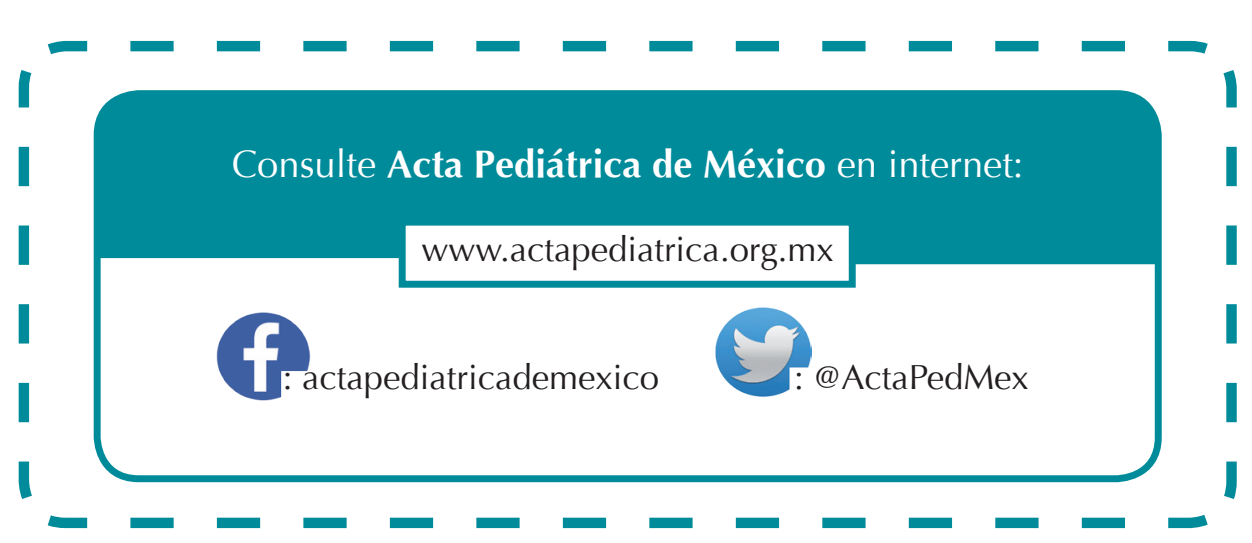

